\title{
Neutrino and Positron Constraints on Spinning Primordial Black Hole Dark Matter
}

\author{
Basudeb Dasgupta $\odot^{1, *}$ Ranjan Laha $\odot^{2, \dagger}$ and Anupam Ray $\oplus^{1, \$}$ \\ ${ }^{1}$ Tata Institute of Fundamental Research, Homi Bhabha Road, Mumbai 400005, India \\ ${ }^{2}$ Theoretical Physics Department, CERN, 1211 Geneva, Switzerland
}

(Received 19 December 2019; revised 23 April 2020; accepted 6 August 2020; published 31 August 2020)

\begin{abstract}
Primordial black holes can have substantial spin - a fundamental property that has a strong effect on its evaporation rate. We conduct a comprehensive study of the detectability of primordial black holes with non-negligible spin, via the searches for the neutrinos and positrons in the MeV energy range. Diffuse supernova neutrino background searches and observation of the $511 \mathrm{keV}$ gamma-ray line from positrons in the Galactic center set competitive constraints. Spinning primordial black holes are probed up to a slightly higher mass range compared to nonspinning ones. Our constraint using neutrinos is slightly weaker than that due to the diffuse gamma-ray background, but complementary and robust. Our positron constraints are typically weaker in the lower mass range and stronger in the higher mass range for the spinning primordial black holes compared to the nonspinning ones. They are generally stronger than those derived from the diffuse gamma-ray measurements for primordial black holes having masses greater than a few $\times 10^{16} \mathrm{~g}$.
\end{abstract}

DOI: 10.1103/PhysRevLett.125.101101

Introduction.-Astrophysical observations provide unambiguous evidence of a nonrelativistic, collisionless, and weakly interacting matter, known as dark matter (DM), constituting $\sim 26 \%$ of the total energy density of the Universe [1]. Many well-motivated DM candidates have been proposed and decades of experimental searches conducted, yet the microscopic identity of DM remains unknown. One of the earliest proposed DM candidates is a population of primordial black holes (PBHs) [2-7].

There exist numerous observational constraints on the fraction of DM comprised of PBHs ([8-20], see recent reviews [21-23] and references therein), however, there still exists parameter space where PBHs can form all of the DM [11,24,25]. Multiple ideas have also been proposed in order to probe PBHs in various mass ranges [26-29]. Given this increased scrutiny of PBHs (which started after the direct detection of gravitational waves [30,31] and the subsequent proposal that these $\mathrm{BHs}$ are primordial in nature [32-35]), it is obvious to ask if we have explored all the properties of BHs in our searches for PBHs. Typically, it has been assumed that PBHs have low spins [36-38], however, there exist viable cosmological scenarios where PBHs are born with a high spin [39-46]. Angular momentum is a fundamental property of BHs and it is crucial to explore its implications [47-50]. Here, we study the impact of angular momentum on the observability of PBHs.

Published by the American Physical Society under the terms of the Creative Commons Attribution 4.0 International license. Further distribution of this work must maintain attribution to the author(s) and the published article's title, journal citation, and DOI. Funded by SCOAP ${ }^{3}$.
PBHs with masses $\lesssim 10^{-16} \mathrm{M}_{\odot}$ can be discovered via the observation of particles produced through Hawking radiation. The lifetime of $\mathrm{PBHs}$ with masses less than $2.5 \times 10^{-19} \mathrm{M}_{\odot}\left(3.5 \times 10^{-19} \mathrm{M}_{\odot}\right)$ for nonrotating (maximally rotating) black holes is less than the age of the Universe and it cannot contribute to the DM density [51-54]. The leading constraints on low-mass PBHs arise from the observation of photons [8,55,56], cosmic rays [9], and the $511 \mathrm{keV}$ gamma-ray line [57,58]. Astrophysical observations of neutrinos have been used to constrain particle DM [59,60] and here we study its implications for PBHs. Earlier analyses of the positron and the neutrino observations have focused on nonspinning PBHs [61-67]. Using the latest experimental inputs, we thoroughly investigate the constraints on spinning and nonspinning PBHs.

The origin of the $511 \mathrm{keV}$ gamma-ray line from the Galactic Center (GC) is one of the enduring mysteries of astrophysics [68-71]. Many models have been proposed to explain this observation [72-80], yet none are confirmed. Our constraints are agnostic of these models and robust. The diffuse supernova neutrino background (DSNB) is the accumulation of all neutrinos emitted by core-collapse supernovae over the history of the Universe [81-83]. The current upper limits on the $\bar{\nu}_{e}$ flavor of DSNB are due to the observations in Super-Kamiokande [84], KamLAND [85], and Borexino [86].

We probe the DM fraction of nonspinning and spinning PBHs by considering neutrino and positron emission and possible detection by Super-Kamiokande (Super-K) and INTEGRAL, respectively. By considering a log-normal mass distribution, we can probe further into a previously unexplored mass window. At low masses, our 
results constrain the possibility that $\mathrm{PBHs}$ make up all the DM.

Methods and results.-PBHs can have wide range of masses depending on its formation time [8]. An uncharged rotating $\mathrm{BH}$ radiates with a temperature [51,52,87-89]

$$
T_{\mathrm{PBH}}=\frac{1}{4 \pi G_{N} M_{\mathrm{PBH}}} \frac{\sqrt{1-a_{*}^{2}}}{1+\sqrt{1-a_{*}^{2}}},
$$

where $M_{\mathrm{PBH}}$ denotes the mass of the $\mathrm{PBH}, G_{N}$ is the gravitational constant, $a_{*} \equiv J /\left(G_{N} M_{\mathrm{PBH}}^{2}\right)$ is the reduced spin parameter, and $J$ is the angular momentum of the $\mathrm{PBH}$. For a given $\mathrm{PBH}$ mass, the temperature can vary by orders of magnitude as the $\mathrm{PBH}$ spin approaches its extremal value, $a_{*} \rightarrow 1$.

The number of emitted particles with spin $s$ in the energy interval $E$ and $E+d E$ and in a time interval $d t$ from a $\mathrm{PBH}$ is

$$
\frac{d^{2} N}{d E d t}=\frac{1}{2 \pi} \frac{\Gamma_{s}\left(E, M_{\mathrm{PBH}}, a_{*}\right)}{\exp \left[E^{\prime} / T_{\mathrm{PBH}}\right]-(-1)^{2 s}},
$$

where $\Gamma_{s}$ is the graybody factor $[48,51-53,88-90]$ and $E^{\prime}$ is the total energy of the emitted species including the rotational velocity. For the rest of our analysis, we will use BlackHawk to compute the spectra of the emitted particle [49]. We have checked this emission rate using the semianalytical formulas from Refs. [51-53,88].

As the temperature of a $\mathrm{PBH}$ becomes comparable to the energy of a particle, such a particle is emitted in significant numbers [51-53]. We first focus on the emission of neutrinos from PBHs. In order to derive bounds from neutrinos, we need to take into account the Galactic and extragalactic contribution of PBHs. The Galactic contribution is given by

$F_{\mathrm{Gal}}=\int \frac{d \Omega}{4 \pi} \int d E \frac{d^{2} N}{d E d t} \int d l \frac{f_{\mathrm{PBH}} \rho_{\mathrm{MW}}[r(l, \psi)]}{M_{\mathrm{PBH}}}$,

where $r$ is the galactocentric distance, $\rho_{\mathrm{MW}}(r)$ denotes the DM profile of the Milky Way (MW), $l$ is the distance from the observer, $\psi$ is the angle between the line of sight and the observer, $\Omega$ is the solid angle under consideration, and the fraction of DM composed of PBHs is denoted by $f_{\mathrm{PBH}}$. The upper limit of the line of sight integral, $l_{\max }$, depends on the MW halo size and $\psi$ [91]. We use the NFW and the isothermal DM profiles using the parametrization in Ref. [91]. For the extragalactic contribution, the differential flux integrated over the full sky is $[8,48]$

$F_{\mathrm{EG}}=\left.\iint d t d \tilde{E}[1+z(t)] \frac{f_{\mathrm{PBH}} \rho_{\mathrm{DM}}}{M_{\mathrm{PBH}}} \frac{d^{2} N}{d E d t}\right|_{E=[1+z(t)] \tilde{E}}$,

where the time integral runs from $t_{\min }=1 \mathrm{~s}$, the neutrino decoupling time, to $t_{\max }$, the smaller of the PBH lifetime and age of the Universe. Although the ultralight PBHs are formed much earlier than the neutrino decoupling time, we have taken it as a lower limit of the time integral $\left(t_{\min }\right)$, because neutrinos emitted from PBHs can free stream after neutrino decoupling. Note that, changing this lower limit to smaller values has very little effect on the corresponding upper limit. The average DM density of the Universe at the present time is denoted by $\rho_{\mathrm{DM}}$. We use the cosmological parameters determined by the Planck observations [1].

In addition to a monochromatic mass function for PBHs, we also consider a log-normal mass function, as predicted by many inflation models:

$$
\frac{d N_{\mathrm{PBH}}}{d M_{\mathrm{PBH}}}=\frac{1}{\sqrt{2 \pi} \sigma M_{\mathrm{PBH}}} \exp \left[-\frac{\ln ^{2}\left(M_{\mathrm{PBH}} / \mu_{\mathrm{PBH}}\right)}{2 \sigma^{2}}\right],
$$

where $\mu_{\mathrm{PBH}}$ and $\sigma$ are the average mass and width of the distribution, respectively.

The upper limit on $f_{\mathrm{PBH}}$ is obtained by comparing the total Galactic and extragalactic flux due to PBHs, with the current upper limit on the neutrino flux from different experiments. We find that neutrino experiments looking for the DSNB are able to set interesting constraints. Neutrinos are emitted as mass eigenstates during $\mathrm{PBH}$ evaporation [92]. So, for $T_{\mathrm{PBH}} \gg m_{\nu}$, the $\bar{\nu}_{e}$ flux is approximately equal to that of any one of the mass eigenstates. Current upper limits on the DSNB flux are $2.9 \bar{\nu}_{e} \mathrm{~cm}^{-2} \mathrm{~s}^{-1}$ $\left(139 \bar{\nu}_{e} \mathrm{~cm}^{-2} \mathrm{~s}^{-1}\right)$ in the energy $\left(E_{\bar{\nu}_{e}}\right)$ range of 17.3 to $91.3 \mathrm{MeV}$ (8.3 to $31.8 \mathrm{MeV})$, respectively [84,85]. We find that the Super-Kamiokande and the KamLAND data help us probe the physical region of $f_{\mathrm{PBH}}<1$. We only show the upper limit obtained using the Super-Kamiokande data, as it is stronger at all PBH masses we consider.

Figure 1 shows the upper limits on $f_{\mathrm{PBH}}$ that can be derived from DSNB searches, for various $\mathrm{PBH}$ mass distributions and spins. The left panel shows the constraints for the monochromatic mass distribution, whereas the middle and the right panels show the constraints for a log-normal distribution with $\sigma=0.5$ and 1 , respectively. For all these cases, we choose $a_{*}=0,0.5,0.9$, and 0.9999 and the NFW profile to derive our limits. Since spinning BHs evaporate faster [51-53], the limits for $a_{*}=0.5$ and 0.9 are stronger than the nonspinning cases for the three mass distributions. For $a_{*}=0.9999$, we find that the constraints are typically weaker than for $a_{*}=0.9$. This can be understood by the rapid decrease in $T_{\mathrm{PBH}}$ as $a_{*} \rightarrow 1$ for a fixed value of $M_{\mathrm{PBH}}$. Because of the much smaller temperature, there are fewer neutrinos in the relevant energy interval, giving a weaker constraint. This constraint is very robust and conservative. It depends on the total DM mass of the MW, but not on the DM profile of the MW. Unlike the positron derived constraint, neutrino derived constraint is minimally sensitive to propagation related uncertainties. These can be due to matter effect and uncertainties in the oscillation parameters. 

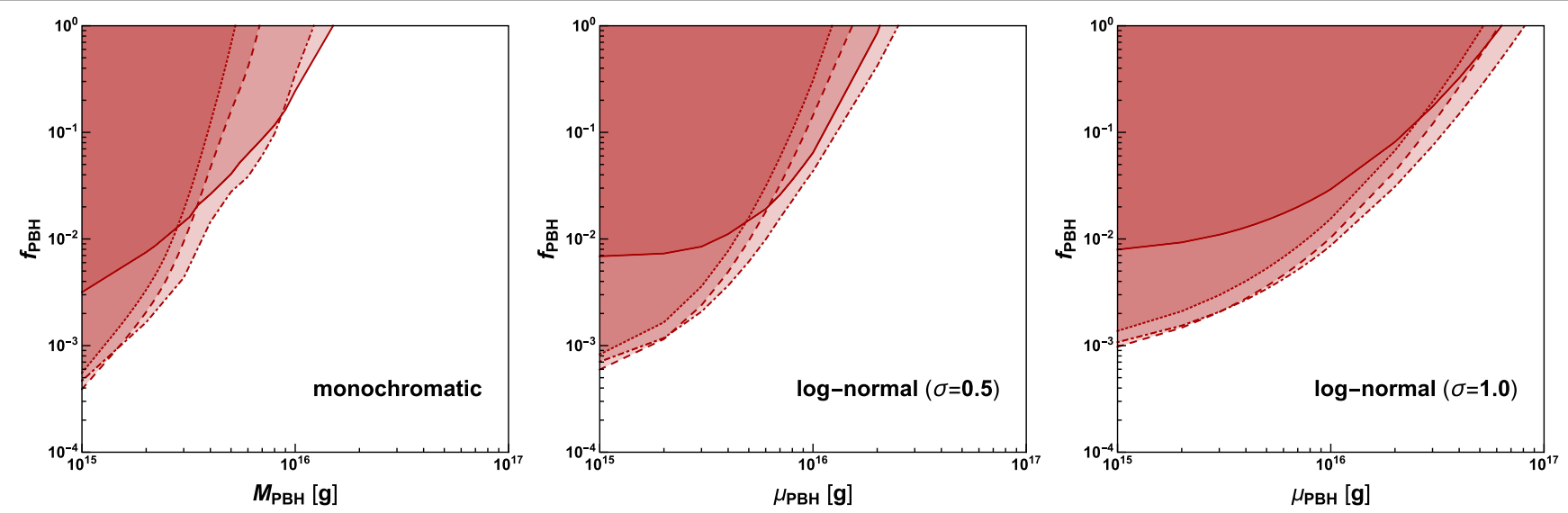

FIG. 1. Upper limit on dark matter fraction of PBHs, $f_{\mathrm{PBH}}$, from DSNB searches at Super-Kamiokande. The left, middle, and right panel corresponds to a monochromatic PBH mass function and log-normal PBH mass functions with $\sigma=0.5$ and 1.0 , respectively. In each plot, four different lines correspond to four different reduced spin parameters $\left(a_{*}=0,0.5,0.9,0.9999\right)$ of PBHs. Tiny dotted, dashed, dot-dashed, and solid lines correspond to $a_{*}=0, a_{*}=0.5, a_{*}=0.9, a_{*}=0.9999$, respectively. These constraints are derived using an NFW dark matter profile of the Milky Way.

For the detection of much lower energy neutrinos, PTOLEMY is a proposed experiment with the capability to detect the cosmic neutrino background [93-96]. We found that the event rate of low energy neutrinos coming from the $\mathrm{PBH}$ evaporation is incredibly small in PTOLEMY and thus it will not be able to set useful limits [92].

The constraints from the GC positrons are derived following Ref. [58]. Given the plethora of astrophysical models to explain the GC $511 \mathrm{keV}$ line, we derive the most conservative bound by simply requiring that the number of positrons injected via $\mathrm{PBH}$ evaporation is smaller than the number of positrons required to explain the observed $511 \mathrm{keV}$ line. The major uncertainty in this technique arises from the unknown propagation distance of positrons in the GC $[72,97,98]$.

The observed flux of $511 \mathrm{keV}$ photons implies that the total production rate of positrons within the Galactic bulge is $\sim 10^{50} \mathrm{yr}^{-1}[70,71,99]$. The limit on the PBH fraction of $\mathrm{DM}\left(f_{\mathrm{PBH}}\right)$ is obtained by requiring that positron injection rate from PBHs obeys this constraint:

$$
f_{\mathrm{PBH}} \leq \frac{10^{50} \mathrm{yr}^{-1}}{\int d E \int d M_{\mathrm{PBH}} \frac{d N_{\mathrm{PBH}}}{d M_{\mathrm{PBH}}} \frac{d^{2} N}{d E d t} \int \frac{d^{3} r \rho_{\mathrm{MW}}(r)}{M_{\mathrm{PBH}}}} .
$$

The energy interval in the above expression runs from 0.511 to $3 \mathrm{MeV}$ [100]. A careful astrophysical modeling of the sources can improve this limit by an order of magnitude [58]. In order to account for the propagation uncertainty of positrons, we consider two different cases: (i) all positrons injected within $1.5 \mathrm{kpc}$ of the GC annihilate to produce the $511 \mathrm{keV}$ signal and (ii) $80 \%$ of positrons injected within $3.5 \mathrm{kpc}$ of the GC annihilate to produce the $511 \mathrm{keV}$ signal $[72,97,98]$.
Figure 2 shows the upper limit on $f_{\mathrm{PBH}}$, from the GC positron observation for various PBH mass functions and reduced spin parameters. The left, middle, and right panels display the constraints for the monochromatic PBH mass function and the log-normal mass distribution with $\sigma=0.5$ and 1, respectively. In each panel, constraints are shown for four different reduced spin parameters, $a_{*}=0,0.5,0.9$, and 0.9999. Because of the semirelativistic nature of the positrons, the constraints for $a_{*}=0$ is the most stringent, especially at low $\mathrm{PBH}$ masses. One can analytically show $\mathrm{PBH}$ spin can lead to $T_{\mathrm{PBH}}<E_{e}$, suppressing positron emission. Because of this suppressed emission, the constraints on $f_{\mathrm{PBH}}$ weaken in parts of the parameter space. Note that the constraints derived from the INTEGRAL observations are stronger than those derived from the diffuse gamma-ray measurements, especially at $\mathrm{PBH}$ masses $\gtrsim$ few $\times 10^{16} \mathrm{~g}$. See Fig. 1 in the Supplemental Material [101] for a more detailed comparison.

Figure 3 shows the variation of the positron constraints for $a_{*}=0.9$, for different DM profiles and propagation distance of low-energy positrons in the GC. Since this variation is a multiplicative constant, as evident from Eq. (6), this uncertainty is the same for PBHs with different spins. The strongest constraint arises when we consider that the DM profile is NFW and that $80 \%$ of positrons injected within $3.5 \mathrm{kpc}$ of the GC annihilate to produce the $511 \mathrm{keV}$ signal. The weakest constraint arises with the isothermal DM profile and a $1.5 \mathrm{kpc}$ region of interest around the GC.

Summary and outlook.-Although PBHs had been written off as the dominant form of DM several years ago, recent studies indicate that such a conclusion was premature. Our constraints represent a valuable contribution to the now ongoing, more careful, reappraisal of the situation. See Fig. 2 in the Supplemental Material [101] for a bird's-eye view of available constraints. Angular 

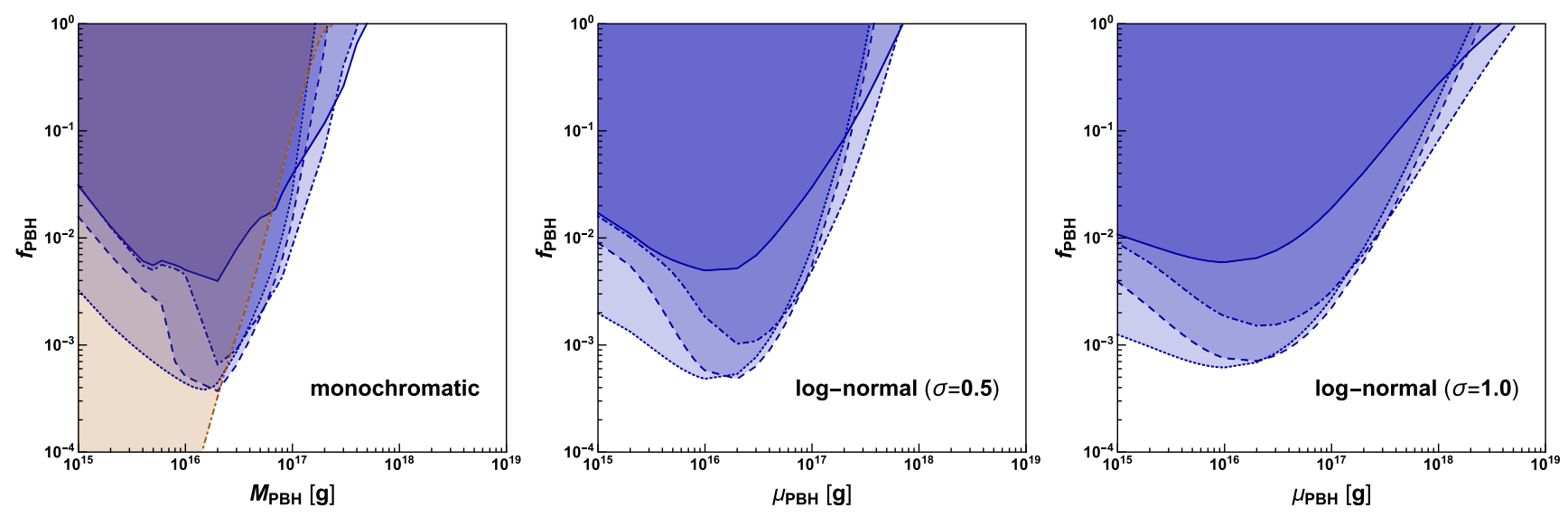

FIG. 2. Upper limit on dark matter fraction of PBHs, $f_{\mathrm{PBH}}$, from INTEGRAL $511 \mathrm{keV}$ gamma-ray line measurement. The left, middle, and right panels correspond to a monochromatic PBH mass function and log-normal PBH mass functions with $\sigma=0.5$ and 1.0 , respectively. In each plot, four different lines correspond to four different reduced spin parameters $\left(a_{*}=0,0.5,0.9,0.9999\right)$ of PBHs. Tiny dotted, dashed, dot-dashed, and solid lines correspond to $a_{*}=0, a_{*}=0.5, a_{*}=0.9, a_{*}=0.9999$, respectively. These constraints are derived using an NFW dark matter profile of the Milky Way and assume that $80 \%$ of positrons within $3.5 \mathrm{kpc}$ of the Galactic center annihilate to produce the $511 \mathrm{keV}$ signal. These constraints are derived using an NFW dark matter profile of the Milky Way and assume that $80 \%$ of positrons within $3.5 \mathrm{kpc}$ of the Galactic Center annihilate to produce the $511 \mathrm{keV}$ signal. In the left panel, the orange line shows the related limit derived from gamma-ray observations [48] for $a_{*}=0.9$. Note that our constraints are more stringent at high mass.

momentum, a fundamental property of BHs, can drastically change the evaporation rate of a $\mathrm{BH}$. There has been a recent surge of interest in spinning PBHs and it is necessary

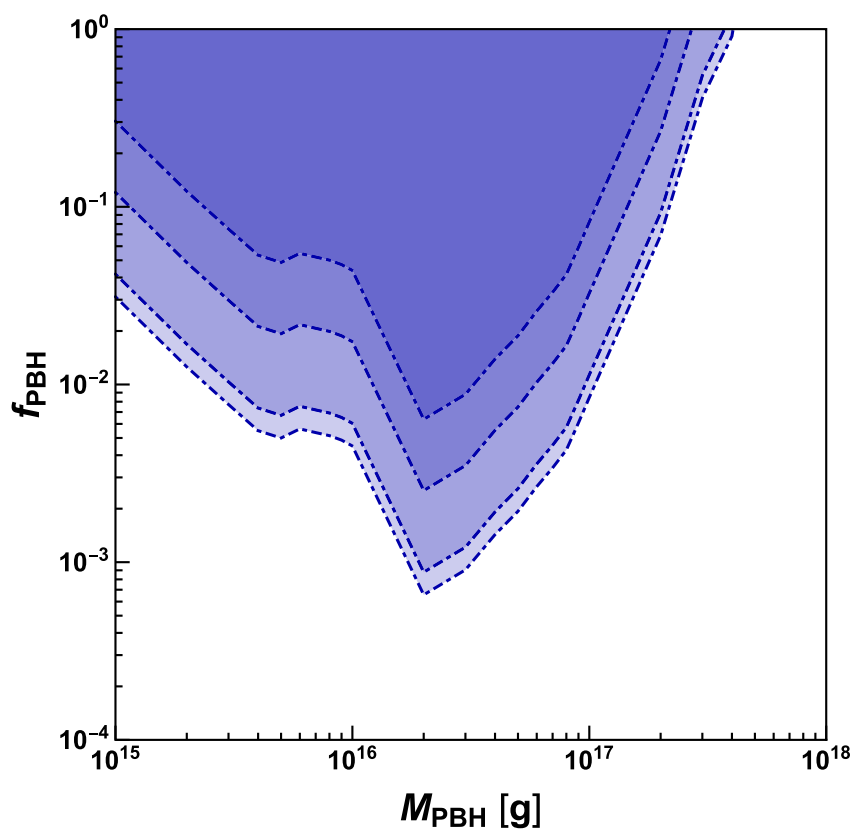

FIG. 3. Variation in the upper limit on dark matter fraction of $\mathrm{PBHs}$ for monochromatic mass distribution from INTEGRAL $511 \mathrm{keV}$ gamma-ray line measurement, due to dark matter density profiles and positron propagation. This plot considers a PBH with $a_{*}=0.9$. The lines from top to bottom correspond to isothermal with $1.5 \mathrm{kpc}$, NFW with $1.5 \mathrm{kpc}$, isothermal with $3.5 \mathrm{kpc}$, and NFW with $3.5 \mathrm{kpc}$ region of interest, respectively. to fully explore the parameter space of these exotic objects. Using DSNB searches and the INTEGRAL observations of the Galactic center $511 \mathrm{keV}$ gamma-ray line, we probe the allowed parameter space of uncharged spinning PBHs. We show that nonzero angular momentum of a $\mathrm{PBH}$ allows us to probe higher mass PBHs. Our constraints using the DSNB (INTEGRAL) observations are weaker (stronger) than the existing limits from the diffuse gamma-ray background. Improved modeling of the GC positrons will also allow us to probe PBHs more comprehensively. These constraints depend on the underlying mass distribution [102-106], however, it is generally true that there exist multiple mass distributions for which PBHs can make up the entire DM density. Although our neutrino constraints are somewhat weaker, they are very robust to uncertainties in the DM density profile (depending only on the total DM mass) and to a variety of astrophysical uncertainties that are inevitably associated with photons or charged particles. Near-future loading of gadolinium in Super-Kamiokande and Hyper-Kamiokande will further enhance their capability to search for the DSNB [107]. Possible DSNB detection, followed by a modeling of the stellar background, will greatly increase the prospect of PBH discovery via neutrinos.

We thank Jeremy Auffinger for help with the BlackHawk package. We are grateful to Jane MacGibbon for pointing us to relevant older literature. The work of B.D. is supported by the Department of Atomic Energy (Govt. of India) research Project No. 12-R\&D-TFR-5.02-0200, the Department of Science and Technology (Govt. of India) through a Ramanujan Fellowship, and by the 
Max-Planck-Gesellschaft through a Max Planck Partner Group. R. L. thanks the CERN Theory Group for support.

*bdasgupta@theory.tifr.res.in

†ranjan.laha@cern.ch

*anupam.ray@theory.tifr.res.in

[1] N. Aghanim et al. (Planck Collaboration), arXiv:1807.06209.

[2] Y. B. Zel'dovich and I. D. Novikov, Astron. Zh. 43, 758 (1966).

[3] S. Hawking, Mon. Not. R. Astron. Soc. 152, 75 (1971).

[4] B. J. Carr and S. W. Hawking, Mon. Not. R. Astron. Soc. 168, 399 (1974).

[5] G. F. Chapline, Nature (London) 253, 251 (1975).

[6] P. Meszaros, Astron. Astrophys. 38, 5 (1975), http:// articles.adsabs.harvard.edu/pdf/1975A\%26A....38....5M

[7] B. J. Carr, Astrophys. J. 201, 1 (1975).

[8] B. J. Carr, K. Kohri, Y. Sendouda, and J. Yokoyama, Phys. Rev. D 81, 104019 (2010).

[9] M. Boudaud and M. Cirelli, Phys. Rev. Lett. 122, 041104 (2019).

[10] H. Niikura et al., Nat. Astron. 3, 524 (2019).

[11] N. Smyth, S. Profumo, S. English, T. Jeltema, K. McKinnon, and P. Guhathakurta, Phys. Rev. D 101, 063005 (2020).

[12] K. Griest, A. M. Cieplak, and M. J. Lehner, Phys. Rev. Lett. 111, 181302 (2013).

[13] R. A. Allsman et al. (Macho Collaboration), Astrophys. J. 550, L169 (2001).

[14] P. Tisserand et al. (EROS-2 Collaboration), Astron. Astrophys. 469, 387 (2007).

[15] L. Wyrzykowski, J. Skowron, S. Kozłowski, A. Udalski, M. K. Szymański, M. Kubiak, G. Pietrzyński, I. Soszyński, O. Szewczyk, K. Ulaczyk et al., Mon. Not. R. Astron. Soc. 416, 2949 (2011).

[16] T. D. Brandt, Astrophys. J. 824, L31 (2016).

[17] Y. Ali-Haïmoud and M. Kamionkowski, Phys. Rev. D 95, 043534 (2017).

[18] M. Raidal, V. Vaskonen, and H. Veermäe, J. Cosmol. Astropart. Phys. 09 (2017) 037.

[19] B. P. Abbott et al. (LIGO Scientific and Virgo Collaborations), Phys. Rev. Lett. 123, 161102 (2019).

[20] M. Zumalacarregui and U. Seljak, Phys. Rev. Lett. 121, 141101 (2018).

[21] B. Carr, K. Kohri, Y. Sendouda, and J. Yokoyama, arXiv:2002.12778.

[22] B. Carr and F. Kuhnel, arXiv:2006.02838.

[23] A. M. Green and B. J. Kavanagh, arXiv:2007.10722.

[24] A. Katz, J. Kopp, S. Sibiryakov, and W. Xue, J. Cosmol. Astropart. Phys. 12 (2018) 005.

[25] P. Montero-Camacho, X. Fang, G. Vasquez, M. Silva, and C. M. Hirata, J. Cosmol. Astropart. Phys. 08 (2019) 031.

[26] S. Jung and T. Kim, Phys. Rev. Research 2, 013113 (2020).

[27] Y. Bai and N. Orlofsky, Phys. Rev. D 99, 123019 (2019).

[28] J. B. Muñoz, E. D. Kovetz, L. Dai, and M. Kamionkowski, Phys. Rev. Lett. 117, 091301 (2016).

[29] R. Laha, Phys. Rev. D 102, 023016 (2020).

[30] B. P. Abbott et al. (LIGO Scientific and Virgo Collaborations), Phys. Rev. Lett. 116, 061102 (2016).
[31] B. P. Abbott et al. (LIGO Scientific and Virgo Collaborations), Astrophys. J. 882, L24 (2019).

[32] S. Bird, I. Cholis, J. B. Muñoz, Y. Ali-Haimoud, M. Kamionkowski, E. D. Kovetz, A. Raccanelli, and A. G. Riess, Phys. Rev. Lett. 116, 201301 (2016).

[33] I. Cholis, E. D. Kovetz, Y. Ali-Haimoud, S. Bird, M. Kamionkowski, J. B. Muñoz, and A. Raccanelli, Phys. Rev. D 94, 084013 (2016).

[34] S. Clesse and J. Garcia-Bellido, Phys. Dark Universe 15, 142 (2017).

[35] M. Sasaki, T. Suyama, T. Tanaka, and S. Yokoyama, Phys. Rev. Lett. 117, 061101 (2016); 121, 059901(E) (2018).

[36] T. Chiba and S. Yokoyama, Prog. Theor. Exp. Phys. 2017, 083E01 (2017).

[37] M. Mirbabayi, A. Gruzinov, and J. Norena, J. Cosmol. Astropart. Phys. 03 (2020) 017.

[38] V. De Luca, V. Desjacques, G. Franciolini, A. Malhotra, and A. Riotto, J. Cosmol. Astropart. Phys. 05 (2019) 018.

[39] M. Y. Khlopov and A. G. Polnarev, Phys. Lett. 97B, 383 (1980).

[40] T. Harada, C.-M. Yoo, K. Kohri, and K.-I. Nakao, Phys. Rev. D 96, 083517 (2017); 99, 069904(E) (2019).

[41] E. Cotner, A. Kusenko, and V. Takhistov, Phys. Rev. D 98, 083513 (2018).

[42] T. Kokubu, K. Kyutoku, K. Kohri, and T. Harada, Phys. Rev. D 98, 123024 (2018).

[43] M. He and T. Suyama, Phys. Rev. D 100, 063520 (2019).

[44] Y. Bai and N. Orlofsky, Phys. Rev. D 101, 055006 (2020).

[45] E. Cotner, A. Kusenko, M. Sasaki, and V. Takhistov, J. Cosmol. Astropart. Phys. 10 (2019) 077.

[46] A. Arbey, J. Auffinger, and J. Silk, Mon. Not. R. Astron. Soc. 494, 1257 (2020).

[47] R. Dong, W. H. Kinney, and D. Stojkovic, J. Cosmol. Astropart. Phys. 10 (2016) 034.

[48] A. Arbey, J. Auffinger, and J. Silk, Phys. Rev. D 101, 023010 (2020).

[49] A. Arbey and J. Auffinger, Eur. Phys. J. C 79, 693 (2019).

[50] F. Kuhnel, Eur. Phys. J. C 80, 243 (2020).

[51] D. N. Page, Phys. Rev. D 13, 198 (1976).

[52] D. N. Page, Phys. Rev. D 14, 3260 (1976).

[53] D. N. Page, Phys. Rev. D 16, 2402 (1977).

[54] B. E. Taylor, C. M. Chambers, and W. A. Hiscock, Phys. Rev. D 58, 044012 (1998).

[55] G. Ballesteros, J. Coronado-Blazquez, and D. Gaggero, Phys. Lett. B 808, 135624 (2020).

[56] R. Laha, J. B. Muñoz, and T. R. Slatyer, Phys. Rev. D 101, 123514 (2020).

[57] W. DeRocco and P. W. Graham, Phys. Rev. Lett. 123, 251102 (2019).

[58] R. Laha, Phys. Rev. Lett. 123, 251101 (2019).

[59] B. Dasgupta and R. Laha, Phys. Rev. D 86, 093001 (2012).

[60] K. Murase, R. Laha, S. Ando, and M. Ahlers, Phys. Rev. Lett. 115, 071301 (2015).

[61] P. N. Okele and M. J. Rees, Astron. Astrophys. 81, 263 (1980), http://articles.adsabs.harvard.edu/pdf/1980A\% 26A....81..2630.

[62] P. Okeke, Astrophys. Space Sci. 71, 371 (1980), http:// articles.adsabs.harvard.edu/pdf/1980Ap\%26SS..71..371O. 
[63] J. H. MacGibbon and B. J. Carr, Astrophys. J. 371, 447 (1991).

[64] C. Bambi, A. D. Dolgov, and A. A. Petrov, Phys. Lett. B 670, 174 (2008); 681, 504(E) (2009).

[65] E. V. Bugaev and K. V. Konishchev, Phys. Rev. D 65, 123005 (2002).

[66] E. V. Bugaev and K. V. Konishchev, arXiv:astro-ph/ 0202519.

[67] E. V. Bugaev and K. V. Konishchev, Phys. Rev. D 66, 084004 (2002).

[68] W. N. Johnson III and R. C. Haymes, Astrophys. J. 184, 103 (1973).

[69] J. Knodlseder et al., Astron. Astrophys. 441, 513 (2005).

[70] N. Prantzos et al., Rev. Mod. Phys. 83, 1001 (2011).

[71] T. Siegert, R. Diehl, G. Khachatryan, M. G. H. Krause, F. Guglielmetti, J. Greiner, A. W. Strong, and X. Zhang, Astron. Astrophys. 586, A84 (2016).

[72] A. Alexis, P. Jean, P. Martin, and K. Ferriere, Astron. Astrophys. 564, A108 (2014).

[73] T. Totani, Publ. Astron. Soc. Jpn. 58, 965 (2006).

[74] P. Sizun, M. Casse, and S. Schanne, Phys. Rev. D 74, 063514 (2006).

[75] R. M. Crocker et al., Nat. Astron. 1, 0135 (2017).

[76] C. Venter, A. Kopp, A. K. Harding, P. L. Gonthier, and I. Busching, Astrophys. J. 807, 130 (2015).

[77] N. Prantzos, Astron. Astrophys. 449, 869 (2006).

[78] G. Weidenspointner et al., Nature (London) 451, 159 (2008).

[79] G. S. Bisnovatyi-Kogan and A.S. Pozanenko, arXiv: 1612.02316.

[80] Y. Farzan and M. Rajaee, J. High Energy Phys. 12 (2017) 083.

[81] S. Ando and K. Sato, New J. Phys. 6, 170 (2004).

[82] J. F. Beacom, Annu. Rev. Nucl. Part. Sci. 60, 439 (2010).

[83] C. Lunardini, Astropart. Phys. 79, 49 (2016).

[84] K. Bays et al. (Super-Kamiokande Collaboration), Phys. Rev. D 85, 052007 (2012).

[85] A. Gando et al. (KamLAND Collaboration), Astrophys. J. 745, 193 (2012).

[86] M. Agostini et al. (Borexino Collaboration), arXiv: 1909.02422.
[87] S. W. Hawking, Commun. Math. Phys. 43, 199 (1975).

[88] J. H. MacGibbon and B. R. Webber, Phys. Rev. D 41, 3052 (1990).

[89] J. H. MacGibbon, B. J. Carr, and D. N. Page, Phys. Rev. D 78, 064043 (2008).

[90] J. H. MacGibbon, Phys. Rev. D 44, 376 (1991).

[91] K. C. Y. Ng, R. Laha, S. Campbell, S. Horiuchi, B. Dasgupta, K. Murase, and J. F. Beacom, Phys. Rev. D 89, 083001 (2014).

[92] C. Lunardini and Y.F. Perez Gonzalez, J. Cosmol. Astropart. Phys. 08 (2020) 014.

[93] S. Weinberg, Phys. Rev. 128, 1457 (1962).

[94] A. G. Cocco, G. Mangano, and M. Messina, J. Cosmol. Astropart. Phys. 06 (2007) 015.

[95] A. J. Long, C. Lunardini, and E. Sabancilar, J. Cosmol. Astropart. Phys. 08 (2014) 038.

[96] M. G. Betti et al. (PTOLEMY Collaboration), J. Cosmol. Astropart. Phys. 07 (2019) 047.

[97] J. C. Higdon, R. E. Lingenfelter, and R. E. Rothschild, Astrophys. J. 698, 350 (2009).

[98] F. H. Panther, Galaxies 6, 39 (2018).

[99] G. M. Fuller, A. Kusenko, D. Radice, and V. Takhistov, Phys. Rev. Lett. 122, 121101 (2019).

[100] J. F. Beacom and H. Yuksel, Phys. Rev. Lett. 97, 071102 (2006).

[101] See Supplemental Material at http://link.aps.org/ supplemental/10.1103/PhysRevLett.125.101101 for comparison of our results with other existing constraints.

[102] B. Carr, F. Kuhnel, and M. Sandstad, Phys. Rev. D 94, 083504 (2016).

[103] B. Carr, M. Raidal, T. Tenkanen, V. Vaskonen, and H. Veermäe, Phys. Rev. D 96, 023514 (2017).

[104] A. M. Green, Phys. Rev. D 94, 063530 (2016).

[105] F. Kuhnel and K. Freese, Phys. Rev. D 95, 083508 (2017).

[106] N. Bellomo, J. L. Bernal, A. Raccanelli, and L. Verde, J. Cosmol. Astropart. Phys. 01 (2018) 004.

[107] J. F. Beacom and M. R. Vagins, Phys. Rev. Lett. 93, 171101 (2004). 